\title{
Extrinsic duodenal obstruction by congenital bands in an adult patient
}

\author{
G.E. Górnacz and A.M. Lewis
}

Royal Free Hospital, Pond Street, London NW3 2QG, UK.

\begin{abstract}
Summary: An adult patient with congenital malrotation of the gut, presenting as duodenal obstruction is reported.
\end{abstract}

\section{Introduction}

A 64 year old man who developed extrinsic duodenal obstruction due to congenital bands associated with midgut malrotation is presented. Although scanty reports of malrotation presenting in adults exist, a search of the literature has revealed only two previous cases associated with transduodenal bands, both occurring in much younger patients.

\section{Case report}

A 64 year old car production supervisor, presented in out-patients with a six month history of episodic vomiting, distension, and weight loss of 2 stones. There was no relevant past history. Physical examination was unremarkable but his barium meal showed considerable gastric stasis, and an obstruction at the third part of the duodenum as well as malrotation of the intestines (Figure 1).

At laparotomy, the stomach and first two parts of the duodenum were massively dilated, but the third part was obstructed by strong fibrous bands which crossed it anteriorly, tethering the right colon to the posterior abdominal wall - the bands of Ladd. There was a high mobile midline caecum, the large bowel was entirely on the left side of the abdomen, and the small intestine on the right side. There was no evidence of volvulus. Simple division of the bands was performed following which the patient made an uneventful recovery. A subsequent barium meal showed malrotation was still present, but there was now no evidence of intestinal obstruction (Figure 2).

G.E. Górnacz F.R.C.S. and A.M. Lewis, F.R.C.S Accepted: 13 December 1983

\section{Discussion}

Duodenal obstruction by congenital bands is extremely rare in adults and a search of the literature revealed only 2 previous cases (Devlin, 1971; Wang \& Welch, 1963). There are also scanty reports of midgut malrotation presenting in adult life. In the newborn, malrotation is almost always accompanied by high intestinal obstruction, resulting from compression of the duodenum by Ladd's bands (Ladd, 1932). These are strong condensations of parietal peritoneum pass-

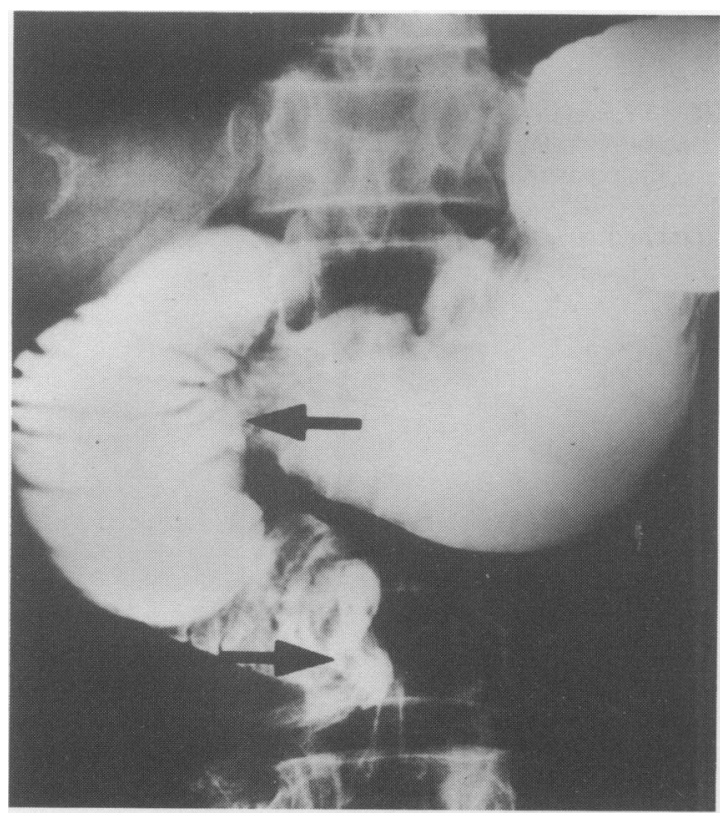

Figure 1 Pre-operative barium meal. Arrows indicate the grossly distended duodenum (upper arrow) and the site of obstruction in its third part (lower arrow).

(C) The Fellowship of Postgraduate Medicine, 1985 


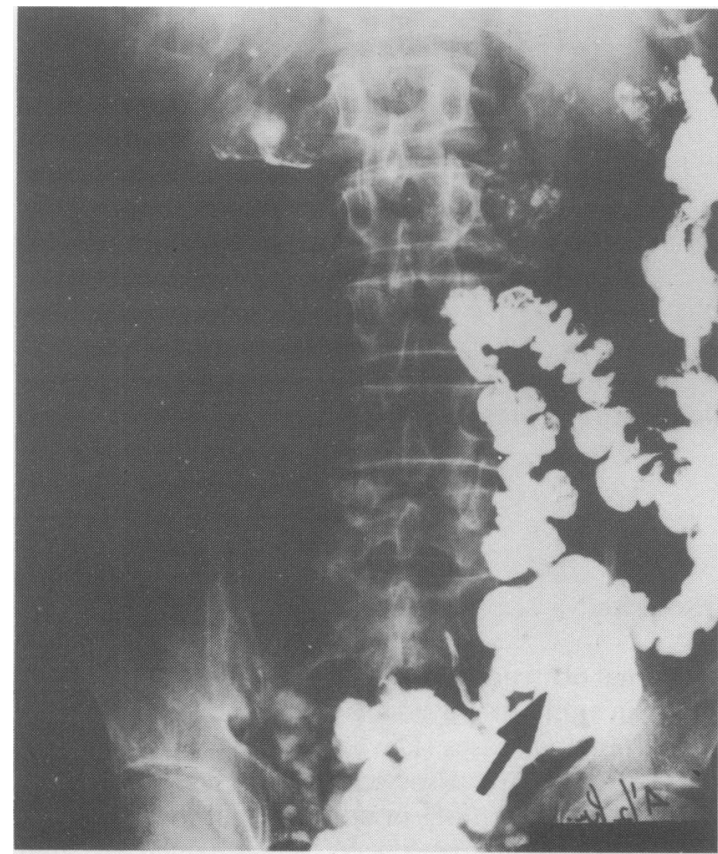

Figure 2 Post-operative barium meal and follow through. Late film showing barium in the colon which is seen to lie entirely to the left of the midline. The arrow indicates the caecum.

\section{References}

DEVLIN, B. (1971). Midgut malrotation causing intestinal obstruction in adult patients. Annals of the Royal College of Surgeons of England, 48, 227.

LADD, W.E. (1932). Congenital obstruction of the duodenum in children. New England Journal of Medicine, 206, 277. ing from the abnormally placed caecum, laterally气 across the duodenum to the posterior abdominal wall. 3

Snyder and Chaffin (1954) reported 40 cases of neo- $\stackrel{\odot}{\complement}$ natal malrotation seen at Los Angeles Children's $C$ Hospital, all of which also had high intestinal obstruc- $\overrightarrow{\vec{F}}$ tion either due to Ladd's bands or to involvement of $\stackrel{\text { ? }}{+}$ the duodenum at the base of a midgut volvulus. Devlin (1971) and Wang and Welch (1963) each described a 음 similar case to the present one in girls aged 14 and $18 \frac{\pi}{\sigma}$ respectively, both with congenital bands. The present $\propto$ case appears to be the third described in an adult (and $\%$ certainly the oldest) and illustrates the need to con- $\vec{\circ}$ sider anomalies of rotation in the differential diagnosis of duodenal obstruction even after the neonatal $\vec{\omega}$ period.

\section{Acknowledgements}

We thank Mr R. Ramsay, M.B.E. for allowing us to present his patient, and Miss D. Noyek for typing the manuscript.

SNYDER, W.H. \& CHAFFIN, L. (1954). Embryology and pathology of the intestinal tract - presentation of 40 cases of malrotation. Annals of Surgery, 140, 368.

WANG, C. \& WELCH, C. (1963). Anomalies of intestinal rotation in adolescents and adults. Surgery, 54, 839. 